\title{
The Study of Intelligent Scheduling Algorithm in the Vehicle ECU based on CAN Bus
}

\author{
Yi Wang ${ }^{1, *}$, Lin-hu Hui ${ }^{1}$ and Biao Liu $^{2}$ \\ ${ }^{I}$ School of Physics and Electronics Science, Guizhou Normal University, Guiyang, 550001, P.R. China, ${ }^{2}$ School of Elec- \\ trical Engineering, Beijing Jiaotong University, Beijing 100044, P.R. China
}

\begin{abstract}
In this paper, a new intelligent scheduling algorithm, which can improve the network utilization rate, had been present based on analyzing the vehicle CAN bus network schedulability in the vehicle CAN bus electrical control system. Through the mathematical modeling of the vehicle CAN bus network control system, simulation modeling of the 3-loop CAN bus system and computer simulation research based on TrueTime, the results proved that the intelligent scheduling algorithm compared with the existing static or dynamic scheduling algorithms could effectively schedule the various data in the vehicle electric control system based on CAN bus, and improve the system performance. The research results have the reference value in the effective algorithm study for the control system of vehicle CAN bus network.
\end{abstract}

Keywords: CAN bus, network utilization rate, scheduling algorithm, TrueTime simulating.

\section{INTRODUCTION}

CAN bus is the most successful vehicle network standard and widely used in the vehicle control system [1-3]. Due to the development of technology and improve demands about the vehicle control performance, comfortableness and safety performance, ECU (Electronic Control Units) have increased sharply and thereupon leads to the increase of the data transmitted and shared [4]. Therefore, there are more and more challenges about real-time performance, effectiveness and bandwidth resource utilization rate in the data transmitting of the vehicle ECU based on CAN bus $[5,6]$. A reasonable scheduling algorithm is needed to make the fully use of the vehicle CAN bus network under the insurance of favorable QoC (Quality of Control) and QoS (Quality of Service) of the vehicle ECU based on CAN bus [7-9]. In this article, by taking the vehicle ECU based on CAN bus as the research object, though the researching and analyzing about the schedulability and scheduling algorithms of the vehicle CAN bus network, the ISA (Intelligent Scheduling Algorithm) had been present, then CAN bus control system had been simulated by using the TrueTime network toolbox, it was proved that the ISA is correct and effective.

\section{SCHEDULABILITY RESEARCH OF THE VEHI- CLE CAN BUS NETWORK}

The purpose of the schedulability research of the vehicle CAN bus network is to compel the predictability and manageability in the data transmission of vehicle network, and then allocates the network resources rationally. The CAN network is band-limited, and the maximal communication rate could only reach $1 \mathrm{M}$ bps. Therefore, taking fully use of network bandwidth, utilizing data rationally and controlling the network load can improve the network running performance and effectively reduce the problems as data transmission delay, data-packet dropout and etc. At present, however, the widely used method in the scheduling algorithms research is the response-time scheduling algorithms, which schedules the system by analyzing the maximal response time and then comparing with the end time of each task.

$t_{R m}$, the longest waiting time in the data transmission, is an important parameter to determine whether a vehicle CAN bus could be scheduled or not. It is defined as the longest time required from data enters the sending notes transmission queue till to it has been received correctly by the target notes [10]. Formulated as

$t_{R m}=t_{J m+} t_{I m}+t_{C m}$

In which: $t_{J m}$-the software perturbation time of sending data $S_{m}$

$$
\begin{aligned}
& t_{I m} \text {-the blocked time of } S_{m} \\
& t_{C m} \text {-the transmission time of } S_{m}
\end{aligned}
$$

The schedulability of the vehicle CAN bus network is to find out the relationship between the longest waiting time $t_{R m}$ and the deadline $t_{D m}$ of the data $S_{m}$ to insure the real-time in the CAN bus data transmission, data $S_{m}$ in the transmission queue should be send before next data $S_{m}{ }_{m}$ come into that queue, otherwise, data $S_{m}$ will be placed by data $S_{m}{ }_{m}$, and data will be lost in the transmission. To any data $S_{m}$, if it satisfy the following formula

$t_{R m}<t_{D m}-t_{I m}$

In which, $t_{D m}$ is the deadline of transmission data.

The CAN bus data transmission is real-time and the network is schedulability [11]. The purpose to choice the 
scheduling algorithm is to insure the schedulability of the network.

\section{RESEARCH OF ISA (INTELLIGENT SCHEDUL- ING ALGORITHM)}

The key problem in scheduling the CAN bus network is to harmonize the relationship between resource allocation and control performance. That means to allocate the network resources rationally for each kind of data in sequence, and satisfy the performance requirements for the whole network control system. The present scheduling algorithms mainly used in vehicle CAN bus network were static scheduling algorithms and dynamic scheduling algorithms [12]. In the static scheduling algorithms, sampling period or priority will be allocated off-line, and not change in the system running; in the dynamic scheduling algorithms, time variation of the network data will be considered and sampling period or priority will be allocated on-line according to system requirements. Typical static scheduling algorithms include RM (Rate Monotonic), DM (Deadline Monotonic) and FP (Fixed priority). The static scheduling algorithms are suitable for scheduling the deterministic and hard real-time systems. But they are less flexibility when the data time characteristics change in the transmission, and the resource utilization rate of the communication network is low. The dynamic scheduling algorithms can allocate the priority dynamically to flexibility adapt the change of system, and make a high network resource utilization rate. But there are also some shortcomings, such as: the heavy calculation of system, the complexity of algorithm coding and much more system overhead. Besides, there are MTS (Mixed Traffic Scheduling) [13] and EMTS (Easy Mixed Traffic Scheduling), etc.

The purpose of the intelligent scheduling algorithm research of the vehicle CAN bus network is to compel the predictability and manageability in the data transmission of vehicle network, and then allocates the network resources rationally. A dynamic data scheduler should be designed in order to scheduling the CAN bus data intelligently. The dynamic data scheduler comprises three function modules by the function requirements: network status monitoring module (for getting the current working state information of CAN bus), intelligent scheduling algorithm module (for calculating the next working state of CAN bus automatically and choosing the sampling period of the control system) and network state executing module (for executing the intelligent algorithm and adjusting the system sampling period).

The algorithm procedures are as follow:

Step 1: The network status monitoring module samples the current time $\left(t_{k}\right)$ working state $S$ of the CAN bus network, and calculate the parameters such as CAN bus resource utilization rate $U$, data transmission time $C$ and the control system sampling period $T$ based on it.

Step 2: The intelligent scheduling algorithm calculates the CAN bus resource utilization rate $\bar{U}$, the data transmission time $\overline{\boldsymbol{C}}$ and the control system sampling period $\overline{\boldsymbol{T}}$ of the next time $\left(t_{k+1}\right)$.

Step 3: The intelligent scheduling algorithm consists of two parts: predicting algorithm and sample period adjusting algorithm. Predicting algorithm takes the job of step 2; and sample period adjusting algorithm reallocates the network resource according to $\overline{\boldsymbol{C}}$ and $\overline{\boldsymbol{U}}$.

Step 4: The network state executing module compares the CAN bus resource utilization rate $\overline{\boldsymbol{U}}$ in the next time $\left(t_{k+1}\right)$ with the upmost utilization set rate $U_{s p}$, and adjusts the sampling period according to the intelligent scheduling algorithm to balance the network load.

Using this method to schedule each data in the CAN bus network, the data priority, the data bandwidth occupancy rate, the data sending time and the data deadline will be overall considered. This algorithm is intelligent and can improve the system performance.

\section{MODELING OF THE VEHICLE CAN BUS NET- WORK CONTROL SYSTEM}

Fig. (1) is a vehicle CAN bus network control system. There are $\mathrm{n}$ independent control loops in it and using the CAN bus as the communication medium. The communication protocol is CAN 2.0A/CAN 2.0B. Data transmit in the sensor, controller and actuator. Its characteristics are distributed, real-time, feedback etc. In this type of system, the controlling algorithm will not only influence the control performance of each loop, but also affect to allocate the network

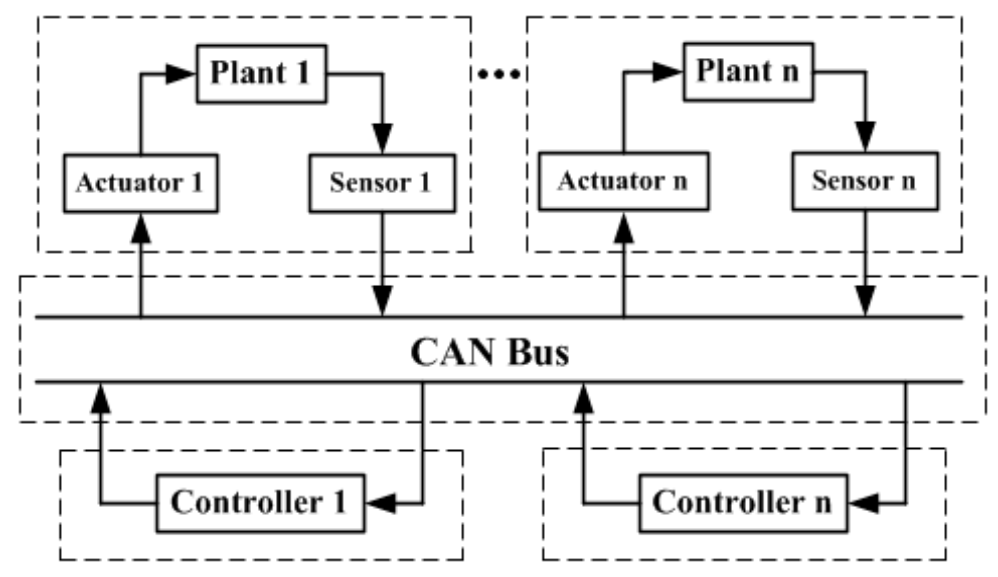

Fig. (1). The structure of vehicle CAN bus network control system. 


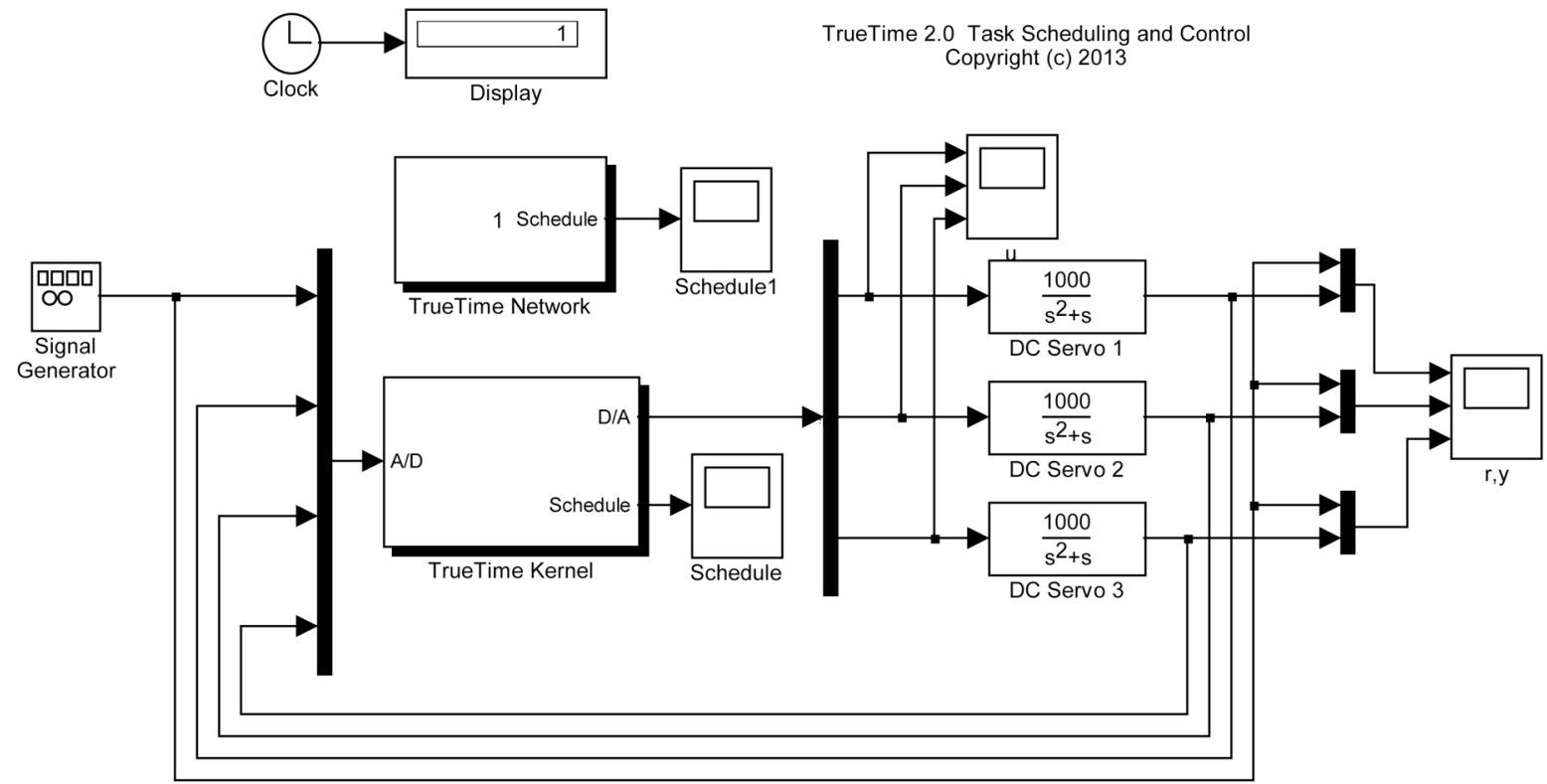

Fig. (2). The simulation structure of CAN bus network control system scheduling.

sharing resources rationally. In the vehicle CAN bus network control system, data in each control loop will transmit by the sensor, controller, actuator and controlled object sequentially. The entire process from the sensor sampling to the actuator acting on the controlled object forms a control system signal flow.

In a vehicle CAN bus network control system with 3 same structure independent control loops, the sensor is timedriven, the controller and actuator are event-driven. Sensor to controller and controller to actuator are communicate by CAN bus network. The state-space representations are as follow [14]:

$\left\{\begin{array}{l}\dot{x}(t)=A x(t)+B u(t) \\ y(t)=C x(t)\end{array}\right.$

In the formula: $\quad x(t) \in R^{n}, \quad u(t) \in R^{r}$, $y(t) \in R^{m}, x(t)$ is the system state variable; $u(t)$ is the system controlled variable; $y(t)$ is the system output variable.

The system transfer function will be as followed based on formula (3)

$G(s)=C(s I-A)^{-1} B$

Take the follow controlled object for example [15]:

$\left\{\begin{array}{l}\dot{x}(t)=\left[\begin{array}{cc}0 & 1 \\ 0 & -1\end{array}\right] x(t)+\left[\begin{array}{l}1 \\ 1\end{array}\right] u(t) \\ y(t)=\left[\begin{array}{ll}1000 & 0\end{array}\right] x(t)\end{array}\right.$

Its transfer function will be as follow:

$G(s)=\frac{1000}{s^{2}+s}$

The simulation model of CAN bus network control system was built by using the TrueTime tool in the Mat-
lab/Simulink simulation platform [16], in which, sensor, controller and actuator were replaced by a Kernel module separately, and the CAN bus network was replaced by Network module. The simulation model has 3 closed loops. Each loop contains sensor, controller and actuator. The simulation model structure is showed as Fig. (2).

\section{SIMULATION RESEARCH}

During simulating the scheduling algorithm, each mode in Fig. (2) shall be initialized and set the information parameter through compiling the $\mathrm{M}$ document. The system parameters can be defined through the setting of the windows by mode (e.g. the selection of the network protocol, the setting of the network rate and the simulation time) The CAN bus network control system in Fig. (2) shall be simulated with the methods of Rate Monotonic, Earliest Deadline First, and the Intelligent Scheduling Algorithm respectively. The sampling cycles of three loops are selected: $h_{I}=0.02$, $h_{2}=0.03, h_{3}=0.04$. The network parameters are set to: the CAN protocol, the $120 \mathrm{Kbps}$ data transmission rate, 0 data loss rate, the maximum utilization rate of the CAN bus network $U_{s p}=80 \%$, and the $0.3 \mathrm{~s}$ simulation time, which can obtain the following timing waveforms of three scheduling algorithms shown as Figs. (3-5). Three points on the longitudinal coordinate indicates the loops, the horizontal ordinate shows the time (Second). In figures, the low level signal means the loop is at the off state with no transmission of the data, the middle level signal indicates the loop is ready for the transmission of the data, and the high level states the transmission of the data.

The figures indicates that the data in the CAN bus network control system can be scheduled with the RM, EDF and ISA scheduling algorithms. But when adopting the ISA, three control loops keep longer at the high level, more data is sent during less time, and the rate of network utilization is more efficient. It indicates that in this control system, the performance of the ISA is superior to the RM and EDF, reduces the conflict during the transmission of the data to 


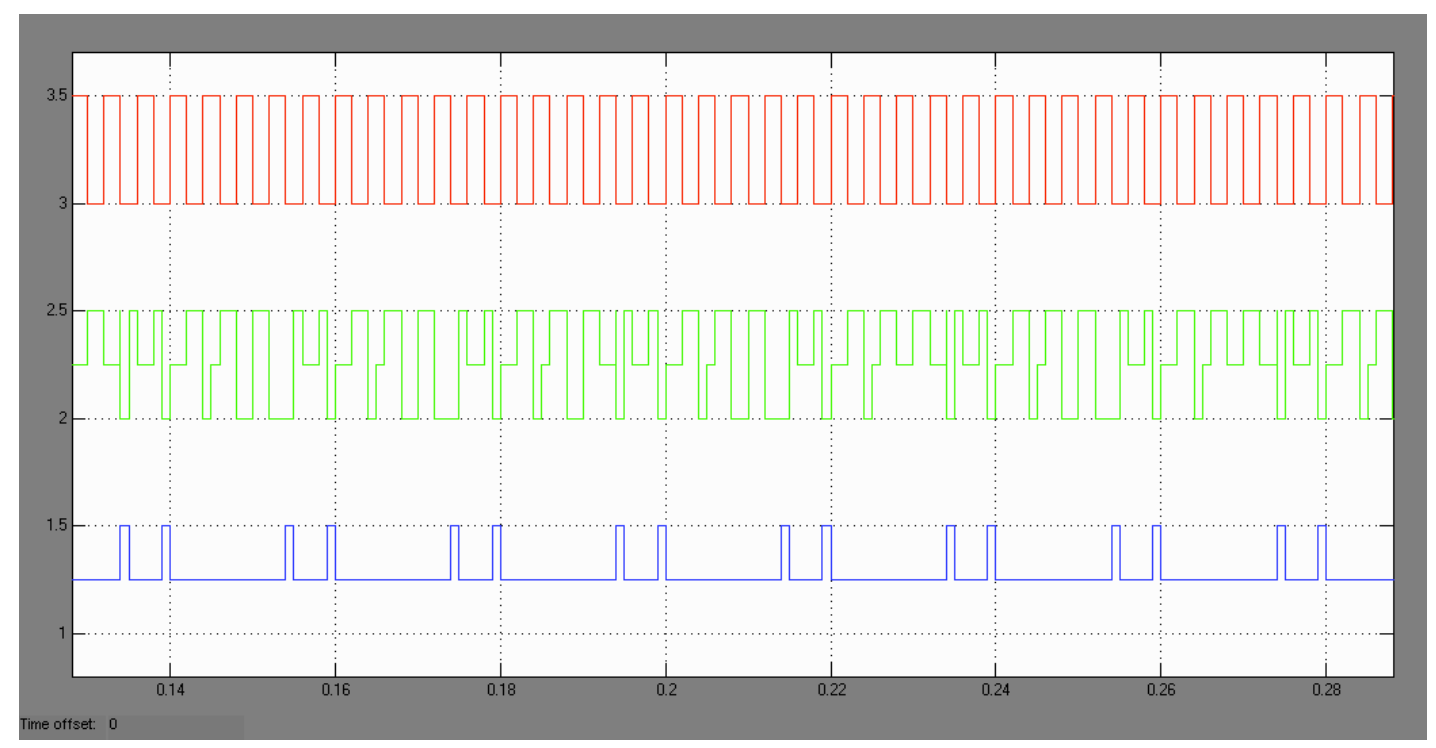

Fig. (3). Waveform of the RM scheduling algorithms.

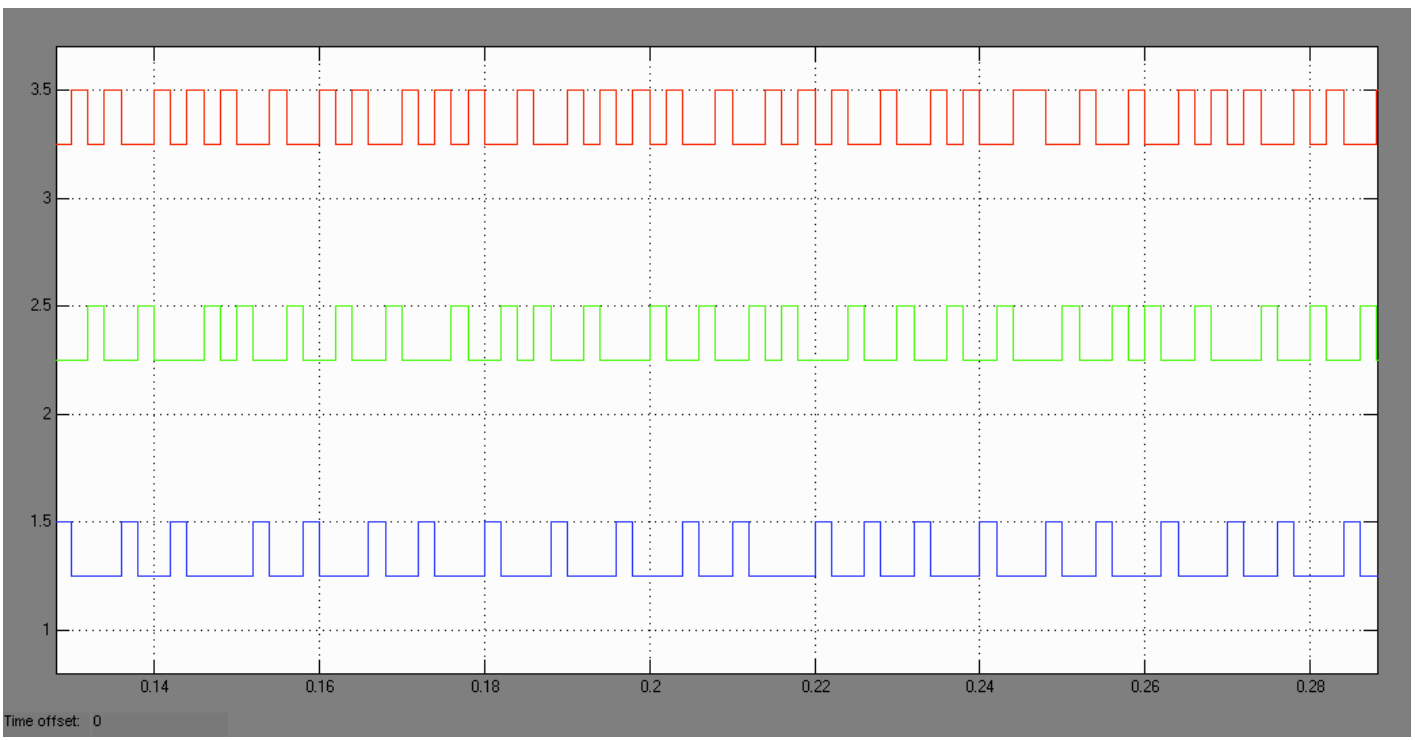

Fig. (4). Waveform of the EDF scheduling algorithms.

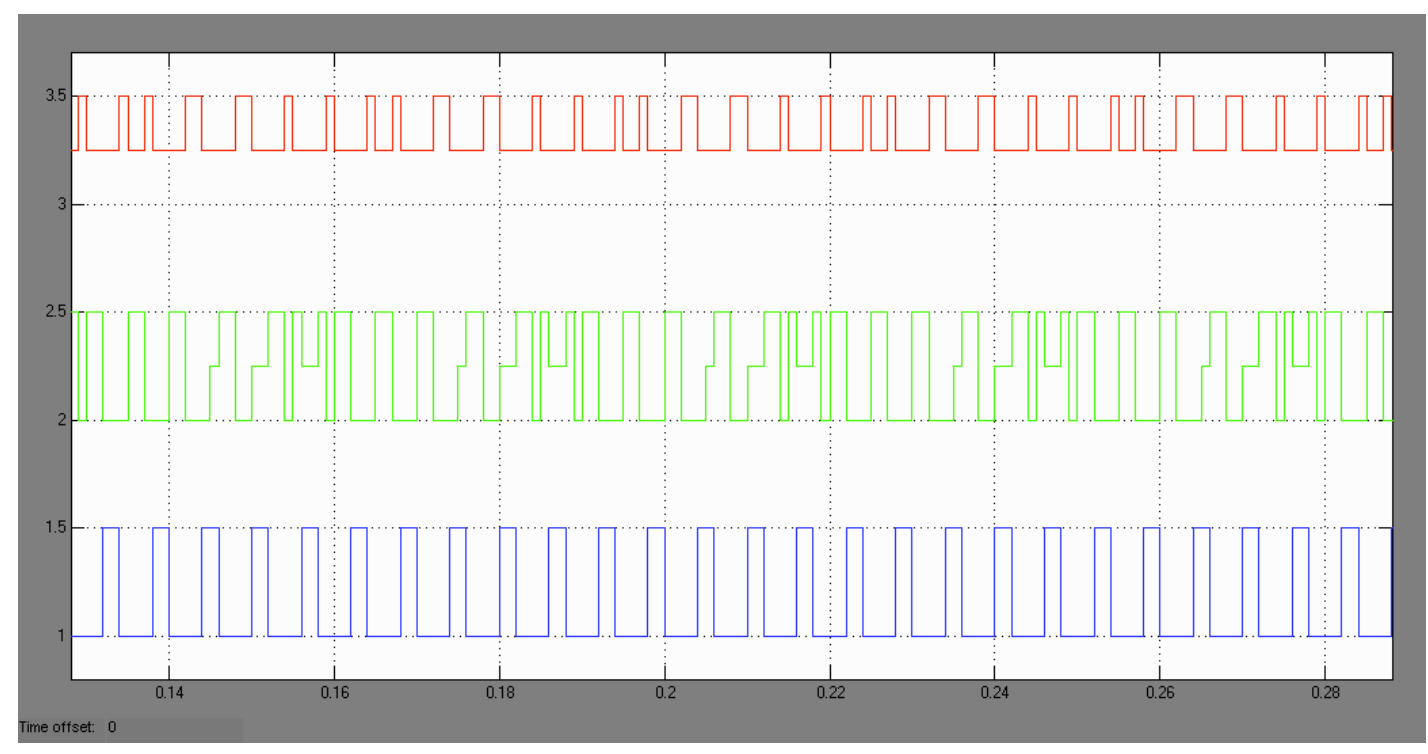

Fig. (5). Waveform of the ISA scheduling algorithms. 
guarantee the rapidity and accuracy, and improve the performance of the CAN bus vehicle network control system.

\section{CONCLUSION}

The CAN bus interconnection structure adopted by the auto electric control system is the key to the networking and intelligence. It has been the most important trend in development of the auto electronic region, and the research involves a wide range area and a complex technology. Especially, the research to the control strategy and scheduling algorithm has great theoretical and practical significance. Considering the relation between the sampling period and the system performance, the paper starts from the research for the schedulability of the CAN bus vehicle network, proposes the ISA (Intelligent Scheduling Algorithm) based on the RM and EDF, establishes the simulation model of the CAN bus network control system and perform the simulation research with Truetime tool. It is verified that the Intelligent Scheduling Algorithm distinguishes from others, which can improve the performance of the control system and the network utilization rate of the auto CAN bus.

\section{CONFLICT OF INTEREST}

The authors confirm that this article content has no conflict of interest.

\section{ACKNOWLEDGEMENTS}

This work was supported by the National Natural Science Foundation of China (Grant No. 61262007 , 61462015), by International Science \& Technology Cooperation Research Foundation of Guizhou Province (Grant No. [2014] 7007), in part by Key Laboratory of Education Department of Guizhou Province (Grant No. KY word [2014] 213).

\section{REFERENCES}

[1] Q. -S. Ye, "Research and application of CAN and LIN bus in automobile network system", In: $3^{\text {rd }}$ International Conference on Advanced Computer Theory and Engineering (ICACTE), Chengdu, China, 2010, pp. 150-154.
[2] W. Yuefei, W. Zihan, and Z. Li, "A new generation automotive bus and relative gateway based on improved scheduling algorithm", Journal of Electronic Measurement and Instrument, vol. 25, no. 10, pp. 894-900, 2011.

[3] C. Zai- ping, and Y. Fang- fang, "Scheduling of real-time control system in CAN network", Control and Instruments in Chemical Industry, vol. 3, no. 40, pp. 346-348, 2013.

[4] H. Jianghong, L. Zhengyu, and C. Shihui, "A study on the application of the controller area network communication protocol to hybrid electric vehicle", Automotive Engineering, vol. 33, no. 12, pp. 1062-1066, 2011.

[5] L. Cheng-zhi, D. Guo-liang, and Y. Liang, "Analysis for real-time performance of improved CAN protocol", Computer Technology and Development, vol. 22, no. 7, pp. 81-84, 2012.

[6] T. Tran, and Q. P. Ha, "Networked control systems with accumulative quadratic constraint", Electronics Letters, vol. 47, no. 2, pp. 108-110, 2011

[7] Y. Sun, and S. Qin, "Stability of networked control systems with packet dropout: an average dwell time approach", IET Control Theory and Applications, vol. 5, no. 1, pp. 47-53, 2011.

[8] Y. F. Wu, G. Buttazzo, E. Bini, and A. Cervin, "Parameter selection for real-time controllers in resource-constrained systems", IEEE Transactions on Industrial Informatics, vol. 6, no. 4, pp. 610$620,2010$.

[9] L. Qi, K. Feng, and Z. Xuelin, "Strategy of time-varying sampling and feedback scheduling for networked control systems", Computer Engineering and Applications, vol. 47, no. 16, pp. 239-241, 2011.

[10] W. Shuju, Z. Tianxia, and Z. Guosheng, "Networks design and schedule strategy of CAN and FTT CAN in vehicle", Transactions of the Chinese Society for Agricultural Machinery, vol. 42, no. 2, pp. 14-17, 2011.

[11] Z. Wei-quan, L. Di, and W. Jia-fu, "Application research on classical scheduling algorithm in networked motion control systems", Computer Engineering and Applications, vol. 46, no. 29, pp. 63-68, 2010.

[12] Y. An-dong, Z. Yun-xiao, and J. Hao, "Research on HEV data communication based on mixed traffic scheduling", Journal of Hefei University of Technology, vol. 35, no. 7, pp. 878-881, 2012.

[13] F. Jinrong, "Optimal cost control for networked control system with variable sampling period", Engineering Journal of Wuhan University, vol. 44, no. 6, pp. 806-810, 2011.

[14] S. Wei- guo, T. Yi, and S. Cheng, "The network control system scheduling algorithm for recursive and variable sampling period", Control and Instruments in Chemical Industry, vol. 38, no. 11, pp. 1299-1302, 2011.

[15] Z. Xiang, and X. Jian, "Analysis and simulation of the networked control system based on truetime", Journal of Lanzhou Jiaotong University, vol. 29, no. 3, pp. 1104-1107, 2010.

\author{
Received: June 10,2015 \\ Revised: July 29, 2015 \\ Accepted: August 15,2015 \\ (c) Wang et al.; Licensee Bentham Open.
}

This is an open access article licensed under the terms of the (https://creativecommons.org/licenses/by/4.0/legalcode), which permits unrestricted, noncommercial use, distribution and reproduction in any medium, provided the work is properly cited. 